\title{
KI-Tetraethylene Glycol Complex as an Effective Catalyst for the Synthesis of Cyclic Thiocarbonates from Epoxides and $\mathrm{CS}_{\mathbf{2}}$
}

\author{
Megumi Okada, ${ }^{[a]}$ Ryuichi Nishiyori, ${ }^{[a]}$ Shiho Kaneko, ${ }^{[a]}$ Kazunobu Igawa, ${ }^{[b]}$ and Seiji Shirakawa*[a]
}

\begin{abstract}
An efficient synthesis of cyclic thiocarbonates from epoxides and $\mathrm{CS}_{2}$ under mild reaction conditions was achieved when a KI-tetraethylene glycol complex was used as a readily available and economical catalyst. The effects of glycols and alkali metal halides were investigated in the present work to clarify the importance of both $\mathrm{KI}$ and tetraethylene glycol. The reaction mechanisms for the cyclic thiocarbonate synthesis are discussed based on the stereochemistry of products.
\end{abstract}

\section{Introduction}

Alkali metal halides, such as potassium and sodium halides, are among the most abundant and economical natural resources. Accordingly, the development of organic reactions using potassium and sodium halide catalysts, which are promoted by utilizing the ionic nature of these salts, is very attractive because it promotes the development of green and sustainable chemistry. ${ }^{[1]}$ However, an alkali metal halide alone is a lessreactive catalyst in organic synthesis, due to its neutrality and to a low level of solubility in organic solvents. To solve these problems and to activate alkali metal halides, polyether compounds, such as crown ethers and polyethylene glycols, are often used with alkali metal halides in organic synthesis. ${ }^{[2]}$ Polyether compounds are known to form complexes with potassium and sodium halides, and these complexes are soluble in organic solvents. Furthermore, the halide anions in these complexes exist in a more naked and nucleophilic version. By utilizing these properties, KI-polyether complex catalysts are applied to $\mathrm{CO}_{2}$ fixation reactions with epoxides 1 , which are important reactions in green and sustainable chemistry. ${ }^{[3-5]}$ The drawback of these catalytic systems has been the harsh reaction conditions (high pressure and high temperature) that are required to promote efficient $\mathrm{CO}_{2}$ fixation. ${ }^{[3 \mathrm{e}, 4,5]}$ In our recent study on the $\mathrm{CO}_{2}$ fixation reactions with epoxides 1 under mild reaction conditions, ${ }^{[6,7]}$ we successfully developed a practical method for

[a] Dr. M. Okada, R. Nishiyori, S. Kaneko, Prof. Dr. S. Shirakawa Department of Environmental Science, Graduate School of Fisheries and Environmental Sciences,

Nagasaki University

1-14 Bunkyo-machi, Nagasaki 852-8521, Japan

E-mail: seijishirakawa@nagasaki-u.ac.jp

Homepage: http://seijishirakawa.wix.com/greenchemistry

[b] Dr. K. Igawa

Institute for Materials Chemistry and Engineering

Kyushu University

Kasuga, Fukuoka 816-8580, Japan

Supporting information for this article is given via a link at the end of the document. the synthesis of cyclic cabonates in the presence of a KItetraethylene glycol complex catalyst (Scheme 1). ${ }^{[8]}$ Based on these findings, we were next interested in the synthesis of cyclic thiocarbonates by the reaction of epoxides 1 with $\mathrm{CS}_{2}$, which is an isoelectronic analogue of $\mathrm{CO}_{2} \cdot{ }^{[9]}$ Although these two reactions are similar, the reaction mechanisms are totally different. In the present article, we discuss and clarify the reaction mechanisms of the cyclic thiocarbonate synthesis based on the stereochemistry of products (Scheme 1)

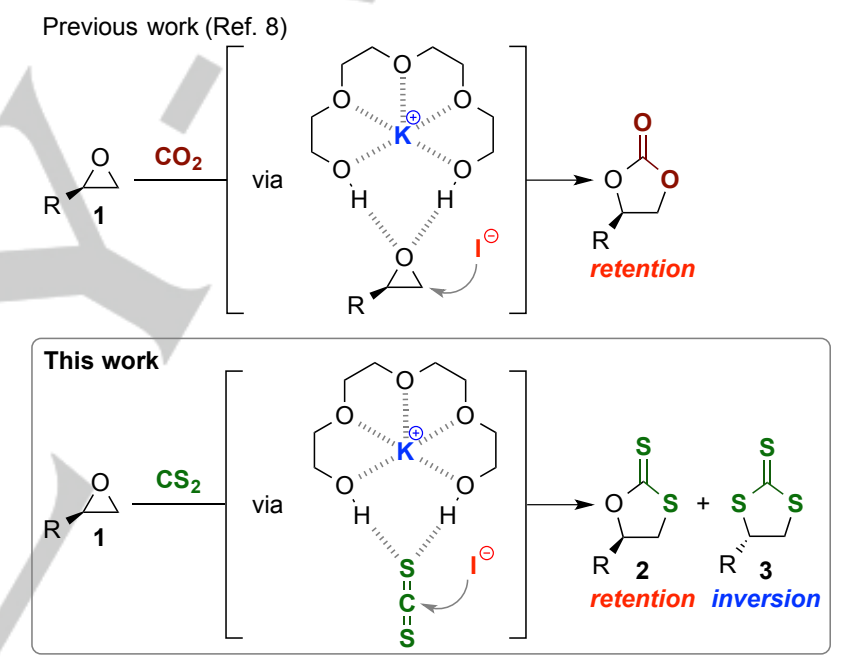

Scheme 1. Synthesis of cyclic carbonates and thiocarbonates with a $\mathrm{KI}-$ tetraethylene glycol complex catalyst.

\section{Results and Discussion}

Our initial aim was to clarify the utility of a KI-tetraethylene glycol complex catalyst in the synthesis of cyclic thiocarbonates under mild conditions. The effects of glycols and alkali metal halides were investigated in a reaction with epoxide $1 \mathrm{a}$ and $\mathrm{CS}_{2}$ (Scheme 2). When a mixture of epoxide $1 \mathrm{a}, \mathrm{CS}_{2}$ (1.2 equiv), and $\mathrm{KI}$ (10 mol \%) was stirred for $5 \mathrm{~h}$ in the absence of tetraethylene glycol at room temperature $\left(25^{\circ} \mathrm{C}\right)$, cyclic dithiocarbonate $2 \mathbf{a}$ was obtained in a low yield (2\%). On the other hand, the $\mathrm{KI}-$ tetraethylene glycol complex catalyst efficiently promoted the reaction of $1 \mathrm{a}$ and $\mathrm{CS}_{2}$ under the same reaction conditions to give product $2 \mathrm{a}$ in a high yield $(88 \%)$. It is noteworthy that cyclic trithiocarbonate $\mathbf{3 a}$ was also isolated as a minor product in this reaction $(4 \%)$. To clarify the role of hydroxy groups in tetraethylene glycol, we also examined the reactions with tetraethylene glycol dimethyl ether and 18-crown-6. Very low 
catalytic activities were observed in these reactions, which gave dithiocarbonate $\mathbf{2 a}$ in low yields with trace amounts of trithiocarbonate $3 \mathbf{a}$. The effect of alkali metal halides was also investigated in the reaction with $\mathrm{CS}_{2}$. Although a $\mathrm{KCl}$ complex with tetraethylene glycol showed quite low reactivity, a $\mathrm{KBr}$ tetraethylene glycol complex catalyst moderately promoted the reaction to give $2 \mathrm{a}$ in a $59 \%$ yield with $3 \mathrm{a}$ in a $2 \%$ yield. The tendency of this reactivity with $\mathrm{KBr}$ is different from the reaction with $\mathrm{CO}_{2}{ }^{[8]}$ The Nal complex showed comparable reactivity in the case of the $\mathrm{KI}$ complex, but the selectivity for dithiocarbonate $\mathbf{2 a}$ to trithiocarbonate $3 a$ was lower $(2 a / 3 a=6.4: 1)$ than that attained with the $\mathrm{KI}$ complex $(2 \mathbf{a} / 3 \mathbf{a}=22: 1)$.
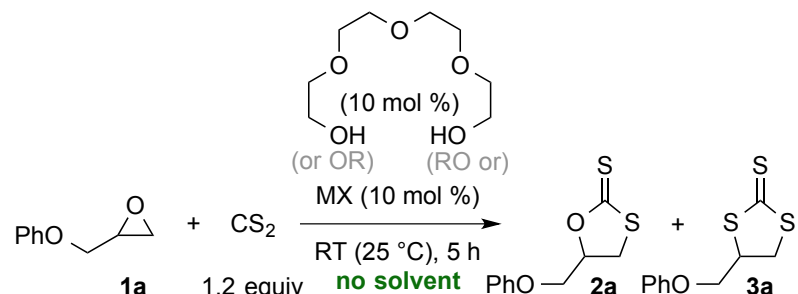
$(4 \mathrm{mmol})$<smiles>CC(C)COCCOCCOCCO</smiles><smiles>COCCOCCOCCOCCOC</smiles><smiles>C1COCCOCCOCCOCCOCCO1</smiles>
$4 \%(3 a)$ $1 \%(3 \mathbf{a})$

\section{$7 \%(2 a)$} trace $(3 a)$<smiles>OCCOCCOCCOCCO</smiles><smiles>OCCOCCOCCOCCO</smiles>

Scheme 2. Effects of glycols and alkali metal halides.

With the effective catalysts in hand, the substrate generality of epoxides 1 for the synthesis of thiocarbonates was examined using a Kl-tetraethylene glycol complex catalyst (Table 1). The reactions were efficiently promoted under mild reaction conditions, and dithiocarbonate products $\mathbf{2}$ were obtained in good-to-high yields. The selectivities for dithiocarbonates 2 to trithiocarbonates 3 were also good-to-high $(2 / 3=4.3: 1->50: 1)$.

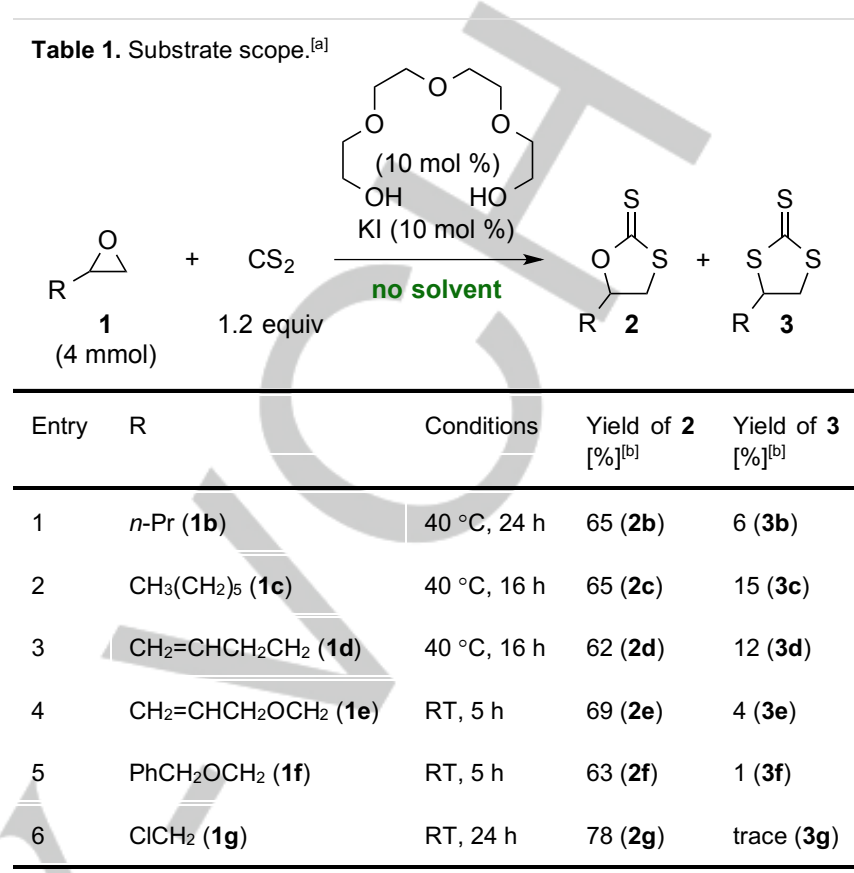

[a] Reaction conditions: 1 (4.0 mmol), tetraethylene glycol $(0.40 \mathrm{mmol}, 10$ $\mathrm{mol} \%), \mathrm{KI}(0.40 \mathrm{mmol}, 10 \mathrm{~mol} \%), \mathrm{CS}_{2}$ (4.8 mmol, 1.2 equiv). [b] Yield of isolated products 2 and 3 .

Enantiopure epoxides (1a, 1f, and $\mathbf{1 h}$ ) were also submitted to the reaction with $\mathrm{CS}_{2}$ under the influence of the KI-tetraethylene glycol complex catalyst (Scheme 3). To our delight, cyclic dithiocarbonates $\mathbf{2 a}, \mathbf{2 f}$, and $\mathbf{2} \mathbf{h}$ were obtained in good yields with a complete "retention" of the stereochemistry. On the other hand, cyclic trithiocarbonates $\mathbf{3 a}, \mathbf{3 f}$, and $\mathbf{3 h}$ were obtained in a complete "inversion" of the stereochemistry. The absolute configurations of the products $\mathbf{2}$ and $\mathbf{3}$ were determined by X-ray diffraction analysis of $\mathbf{2} \mathbf{h}$ and $\mathbf{3} \mathbf{h} .^{[10]}$ Additionally, we proved that Werner's catalytic system that uses a LiOt-Bu catalyst ${ }^{[9 j, k]}$ also showed the same stereochemistry tendency demonstrated in the present reaction. It should be noted that this is a valuable example of the determination of absolute configurations for thiocarbonates in the reaction of enantiopure epoxides and $\mathrm{CS}_{2}$. 


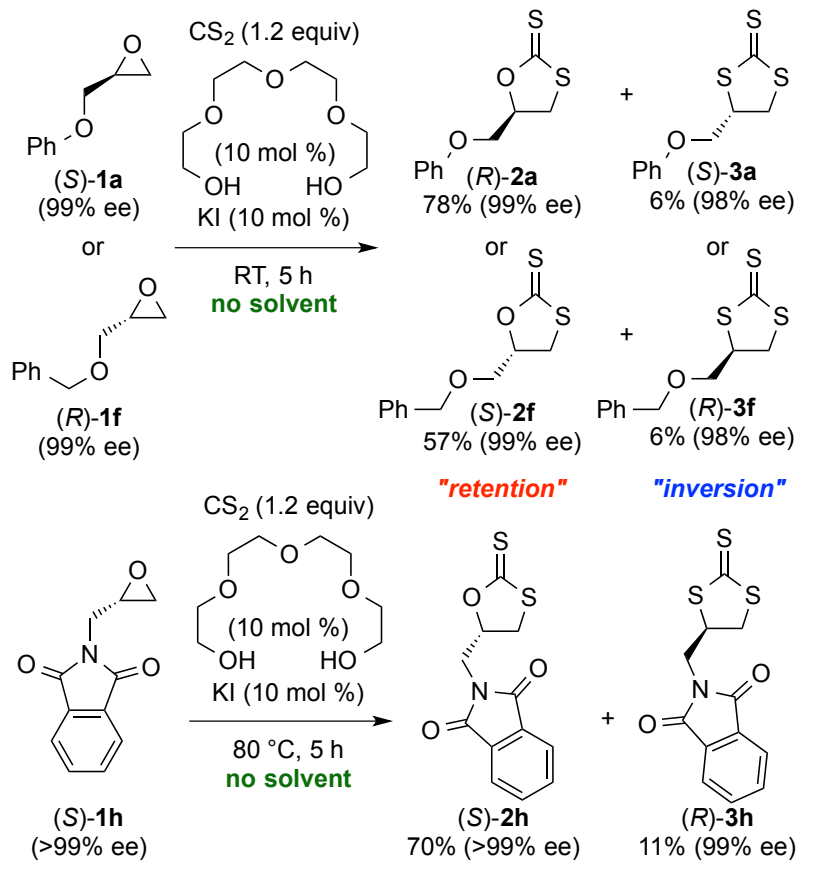

afforded thiirane $\mathbf{4}$ with an inversion of the stereochemistry. ${ }^{[9,111]}$ The reaction of thiirane 4 with $\mathrm{CS}_{2}$ under the influence of a $\mathrm{KI}$ tetraethylene glycol complex gave product 3 in a catalytic cycle similar to that proposed for the reaction with epoxide 1 (Scheme 4). ${ }^{[9, m, 12]}$ Note that trace amounts of thiiranes $\mathbf{4}$ were observed in the crude NMR spectra of several examples in Table 1.

\begin{tabular}{|c|c|c|c|}
\hline \multirow[b]{2}{*}{$\begin{array}{c}(S)-1 h \\
(>99 \% \text { ee })\end{array}$} & $\begin{array}{c}\mathrm{CS}_{2}(2 \text { equiv) } \\
\mathrm{LiOt}-\mathrm{Bu}(10 \mathrm{~mol} \%)\end{array}$ & & \\
\hline & $\underset{\text { no solvent }}{80^{\circ} \mathrm{C}, 5 \mathrm{~h}}$ & $\begin{array}{c}(S)-2 h \\
41 \%(>99 \% \text { ee })\end{array}$ & $\begin{array}{c}(R)-3 \mathbf{h} \\
1 \%(98 \% \text { ee })\end{array}$ \\
\hline
\end{tabular}
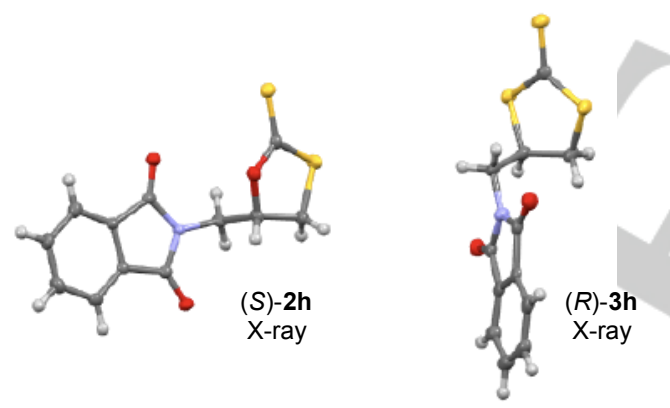

Scheme 3. Reaction with optically active epoxides.

Based on these results and those of previous related reports, ${ }^{[9]}$ the assumed mechanisms for the reaction of epoxides 1 and $\mathrm{CS}_{2}$ were proposed in Schemes 4 and 5. The first step of the reaction with $\mathrm{CS}_{2}$ is known to differ from the reaction with $\mathrm{CO}_{2 .}{ }^{[0]}$ At first, an iodide anion attacks $\mathrm{CS}_{2}$, which was activated via hydrogen-bonding with the hydroxyl groups of tetraethylene glycol (intermediate A). Subsequently, a nucleophilic attack by the resultant iododithioformate anion in intermediate $\mathbf{B}$ on epoxide 1 led to intermediate $\mathbf{C}$. Intramolecular cyclization (intermediate $\mathbf{C}$ ) and the subsequent elimination of the iodide anion (intermediate $\mathbf{D}(\mathbf{1})$ ) led to the attainment of cyclic dithiocarbonate 2 and to the retention of the stereochemistry. On the other hand, cyclic trithiocarbonate $\mathbf{3}$ was obtained via the ring opening in intermediate $\mathbf{D}(2)$ to form intermediate $\mathbf{E}$ (Scheme 5). The intramolecular $\mathrm{S}_{\mathrm{N}} 2$ reaction of the thiolate anion in intermediate $\mathbf{E}$
Scheme 4. Assumed catalytic cycle to produce cyclic dithiocarbonate 2.

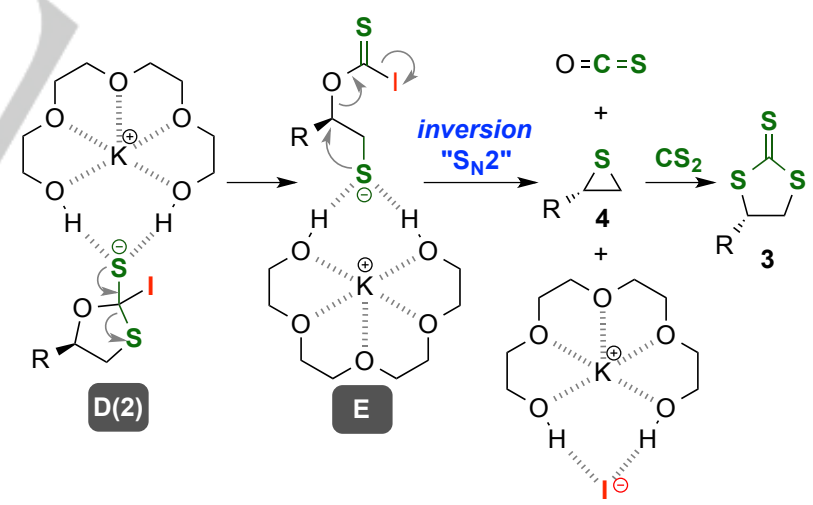

Scheme 5. Proposed mechanism to produce cyclic trithiocarbonate 3.

To expand the utility and further support the mechanism of the present reaction, 1,1- and 1,2-disubstituted epoxides 5 and cis-8 were submitted to the reaction (Scheme 6). The reaction with 1,1-disubstituted epoxide 5 was promoted by the $\mathrm{KI}-$ tetraethylene glycol complex catalyst to give dithiocarbonate $\mathbf{6}$ in a moderate yield $(55 \%)$ with high selectivity (trithiocarbonate 7 : $\sim 0 \%$ ). The reaction with 1,2-disubstituted epoxide cis-8 and $\mathrm{CS}_{2}$ proceeded at room temperature to afford dithiocarbonate trans-9 $(41 \%)$ and trithiocarbonate trans-10 (15\%). ${ }^{[9 b, f, j, k]}$ Notably, the 
reactivity and stereoselectivity were completely different by comparison with the reaction of cis-8 and $\mathrm{CO}_{2}$ (Scheme 7). The reaction with $\mathrm{CO}_{2}$ at $80{ }^{\circ} \mathrm{C}$ gave a cis-11, selectively, via double $\mathrm{S}_{\mathrm{N}} 2$ inversions with activation of epoxide by the catalyst (intermediates $\mathbf{F}$ and $\mathbf{H}$ in Scheme 7 ). In sharp contrast, the product trans-9 was formed as a result of the single $\mathrm{S}_{\mathrm{N}} 2$ inversion in the conversion of intermediate B' to C' (Scheme 8). The trans selectivity of trithiocarbonate 10 could be explained by the formation of thiirane cis-12, which was formed in the same reaction mechanism for the formation of thiirane 4 in Scheme 5. The reaction of thiirane cis-12 with $\mathrm{CS}_{2}$ under the influence of the $\mathrm{KI}$-tetraethylene glycol complex gave trans-10 in a mechanism that was similar to that proposed for the reaction with epoxide cis8 (Scheme 8). These trans selectivities of thiocarbonates 9 and 10 from cis-8 fully support the reaction mechanisms proposed in Schemes 4,5 , and 8.

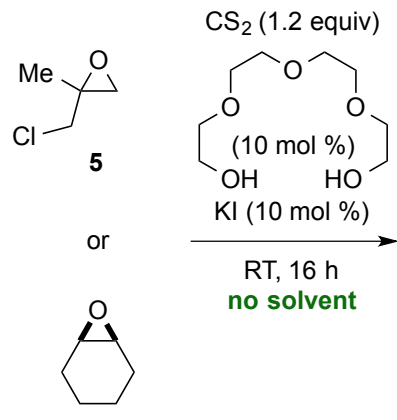

cis-8
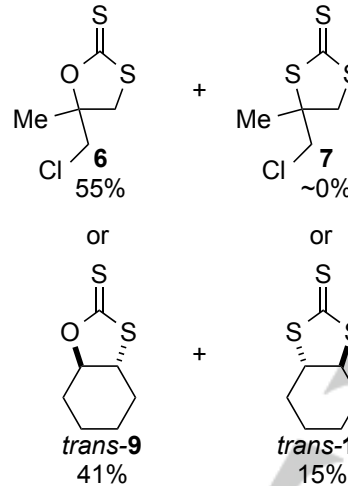

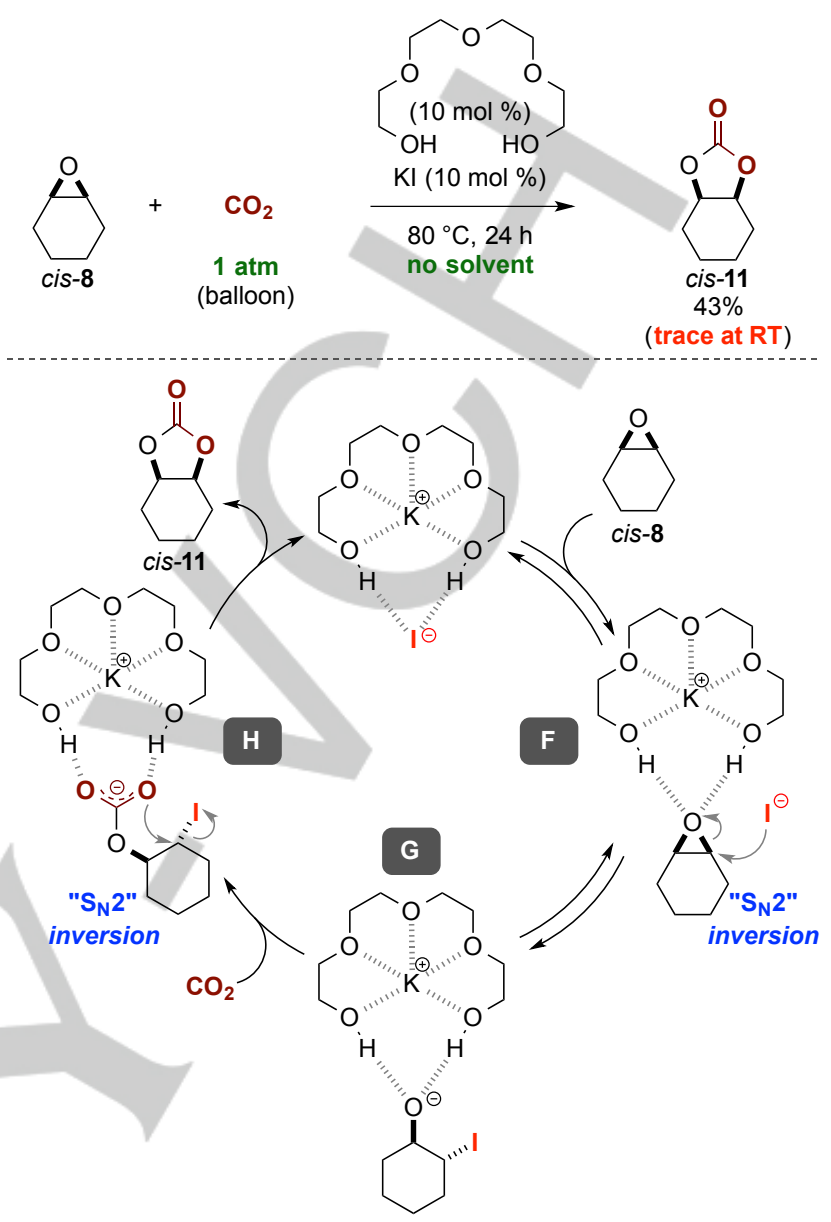

Scheme 6. Reaction with 1,1-disubstituted and 1,2-disubstituted epoxides.

Scheme 7. Reaction with epoxide cis-8 and $\mathrm{CO}_{2}$.

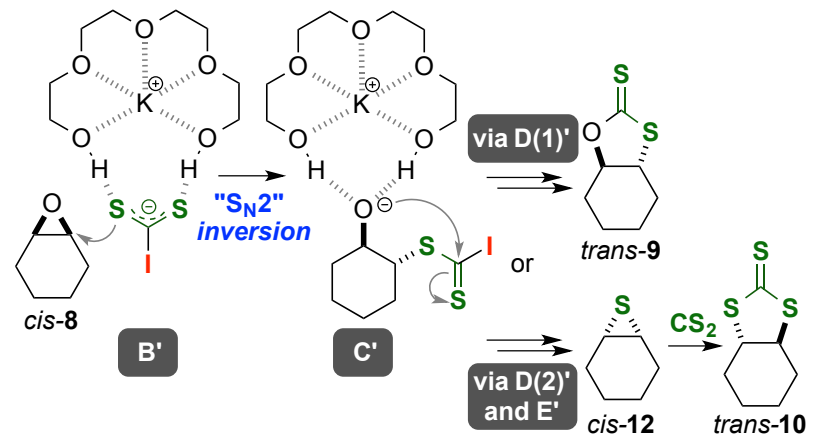

Scheme 8. Proposed mechanism to produce cyclic thiocarbonates $\mathbf{9}$ and $\mathbf{1 0}$

\section{Conclusions}

We have successfully developed an efficient method for the synthesis of cyclic thiocarbonates via a reaction of epoxides and $\mathrm{CS}_{2}$ under mild reaction conditions using a KI-tetraethylene glycol complex catalyst. The effects of glycols and alkali metal halides 
were investigated to clarify the essential points of the catalytic activity. The importance of both the hydroxy groups of tetraethylene glycol and the iodide of the alkali metal halide was clearly observed in the cyclic thiocarbonate synthesis. The reactions of various epoxides and $\mathrm{CS}_{2}$ provided cyclic dithiocarbonates in good yields. In these reactions, cyclic trithiocarbonates were also obtained as a minor product. The optically active epoxides and 1,2-disubstituted epoxides were also submitted to the reactions with $\mathrm{CS}_{2}$ under the influence of a $\mathrm{KI}$-tetraethylene glycol complex catalyst. Although the reaction with $\mathrm{CO}_{2}$ gave cyclic carbonates in retention of the stereochemistry, ${ }^{[8,13]}$ different trends were observed in the reaction with $\mathrm{CS}_{2}$. Based on the stereochemistry of these two reactions, the reaction mechanisms were discussed, and assumed catalytic cycles were proposed. The proposed reaction mechanisms clearly explained the observed stereochemistry of cyclic thiocarbonates, and we concluded that the reactions with $\mathrm{CO}_{2}$ and $\mathrm{CS}_{2}$ proceeded via different mechanisms.

\section{Experimental Section}

Typical procedure for the reaction of epoxides 1 with $\mathrm{CS}_{2}$ catalyzed by a Kl-tetraethylene glycol complex: To a mixture of glycidyl phenyl ether $1 \mathrm{a}(0.542 \mathrm{~mL}, 4.00 \mathrm{mmol})$, tetraethylene glycol $(69.1 \mu \mathrm{L}, 0.400 \mathrm{mmol}$, $10 \mathrm{~mol} \%$ ), and potassium iodide (66.4 $\mathrm{mg}, 0.400 \mathrm{mmol}, 10 \mathrm{~mol} \%$ ) was added $\mathrm{CS}_{2}(0.289 \mathrm{~mL}, 4.80 \mathrm{mmol})$ at room temperature. The reaction mixture was stirred for $5 \mathrm{~h}$ at room temperature $\left(25^{\circ} \mathrm{C}\right)$. The resultant reaction mixture was purified by flash column chromatography on silica gel (hexane/EtOAc $=20: 1-2: 1$ as eluent) to afford cyclic dithiocarbonate $\mathbf{2 a}$ $\left(797 \mathrm{mg}, 3.52 \mathrm{mmol} ; R_{f}=0.42 \mathrm{in}\right.$ hexane/EtOAc $\left.=2: 1\right)$ and trithiocarbonate 3a (38.8 mg, $0.16 \mathrm{mmol} ; R_{f}=0.67$ in hexane/EtOAc $\left.=2: 1\right)$.

2a: ${ }^{[9]}$ Spectral data completely matched with reported data. ${ }^{1} \mathrm{H}$ NMR (400 $\left.\mathrm{MHz}, \mathrm{CDCl}_{3}\right): \delta=7.31(\mathrm{t}, J=8.0 \mathrm{~Hz}, 2 \mathrm{H}), 7.01(\mathrm{t}, J=7.6 \mathrm{~Hz}, 1 \mathrm{H}), 6.92(\mathrm{~d}$ $J=8.8 \mathrm{~Hz}, 2 \mathrm{H}), 5.39-5.46(\mathrm{~m}, 1 \mathrm{H}), 4.24-4.34(\mathrm{~m}, 2 \mathrm{H}), 3.69-3.81(\mathrm{~m}, 2 \mathrm{H})$; ${ }^{13} \mathrm{C} \mathrm{NMR}\left(100 \mathrm{MHz}, \mathrm{CDCl}_{3}\right) \delta=211.2,157.7,129.7,121.9,114.5,87.7$, $66.3,36.3$; IR (neat) 3060, 3038, 2924, 2870, 1597, 1587, 1494, 1240 , $1228,1181,1042,751,689 \mathrm{~cm}^{-1}$.

3a: ${ }^{[9]}$ Spectral data completely matched with reported data. ${ }^{1} \mathrm{H}$ NMR (400 $\left.\mathrm{MHz}, \mathrm{CDCl}_{3}\right): \delta=7.32(\mathrm{t}, J=8.0 \mathrm{~Hz}, 2 \mathrm{H}), 7.02(\mathrm{t}, J=7.6 \mathrm{~Hz}, 1 \mathrm{H}), 6.93(\mathrm{~d}$, $J=8.8 \mathrm{~Hz}, 2 \mathrm{H}), 4.61-4.67(\mathrm{~m}, 1 \mathrm{H}), 4.37(\mathrm{t}, \mathrm{J}=9.6 \mathrm{~Hz}, 1 \mathrm{H}), 4.23(\mathrm{dd}, J=$ $5.8,12.2 \mathrm{~Hz}, 1 \mathrm{H}$ ), 4.20 (dd, $J=5.4,9.8 \mathrm{~Hz}, 1 \mathrm{H}), 4.08(\mathrm{dd}, J=4.0,12.0 \mathrm{~Hz}$, $1 \mathrm{H}) ;{ }^{13} \mathrm{C}$ NMR $\left(100 \mathrm{MHz}, \mathrm{CDCl}_{3}\right) \delta=226.4,157.7,129.7,121.8,114.6$, $66.4,57.2,44.9$; IR (neat) 3046, 1599, 1587, 1496, 1264, 1239, 1078, $1041,732,702,690 \mathrm{~cm}^{-1}$

2b: ${ }^{1} \mathrm{H}$ NMR $\left(400 \mathrm{MHz}, \mathrm{CDCl}_{3}\right): \delta=5.08-5.15(\mathrm{~m}, 1 \mathrm{H}), 3.59(\mathrm{dd}, J=6.6$, $10.8 \mathrm{~Hz}, 1 \mathrm{H}), 3.40(\mathrm{dd}, J=9.4,11.0 \mathrm{~Hz}, 1 \mathrm{H}), 1.98-2.07(\mathrm{~m}, 1 \mathrm{H}), 1.75$ $1.84(\mathrm{~m}, 1 \mathrm{H}), 1.45-1.65(\mathrm{~m}, 2 \mathrm{H}), 1.01(\mathrm{t}, J=7.4 \mathrm{~Hz}, 3 \mathrm{H}) ;{ }^{13} \mathrm{C}$ NMR $(100$ $\left.\mathrm{MHz}, \mathrm{CDCl}_{3}\right) \delta=212.2,91.6,39.3,35.7,18.7,13.7$; IR (neat) 2959, 2932, $2872,1182,1046,830,647 \mathrm{~cm}^{-1}$; HRMS (FAB) $\mathrm{m} / \mathrm{z}$ calcd for $\mathrm{C}_{6} \mathrm{H}_{10} \mathrm{OS}_{2}$ : $162.0173\left(\left[\mathrm{M}^{+}\right]\right)$, found 162.0172 .

3b: ${ }^{1} \mathrm{H}$ NMR $\left(400 \mathrm{MHz}, \mathrm{CDCl}_{3}\right): \delta=4.38-4.45(\mathrm{~m}, 1 \mathrm{H}), 3.97(\mathrm{dd}, J=5.6$, $11.6 \mathrm{~Hz}, 1 \mathrm{H}), 3.71(\mathrm{dd}, J=8.0,12.0 \mathrm{~Hz}, 1 \mathrm{H}), 1.84-2.01(\mathrm{~m}, 2 \mathrm{H}), 1.47$ (sextet, $J=7.6 \mathrm{~Hz}, 2 \mathrm{H}), 0.99(\mathrm{t}, J=7.4 \mathrm{~Hz}, 3 \mathrm{H}) ;{ }^{13} \mathrm{C} \mathrm{NMR}(100 \mathrm{MHz}$, $\left.\mathrm{CDCl}_{3}\right) \delta=228.0,60.7,48.2,35.5,21.5,13.6$; IR (neat) 2956, 2926, 2869, 1461, 1421, 1090, 1049, $874 \mathrm{~cm}^{-1}$; HRMS (FAB) $\mathrm{m} / \mathrm{z}$ calcd for $\mathrm{C}_{6} \mathrm{H}_{10} \mathrm{~S}_{3}$ : $177.9945\left(\left[\mathrm{M}^{+}\right]\right)$, found 177.9945 . 2c: ${ }^{[9]}$ Spectral data completely matched with reported data. ${ }^{1} \mathrm{H}$ NMR $(400$ $\left.\mathrm{MHz}, \mathrm{CDCl}_{3}\right): \delta=5.06-5.14(\mathrm{~m}, 1 \mathrm{H}), 3.58(\mathrm{dd}, J=6.6,11.0 \mathrm{~Hz}, 1 \mathrm{H}), 3.40$ (dd, $J=9.6,11.2 \mathrm{~Hz}, 1 \mathrm{H}), 1.98-2.07(\mathrm{~m}, 1 \mathrm{H}), 1.77-1.86(\mathrm{~m}, 1 \mathrm{H}), 1.25-$ $1.58(\mathrm{~m}, 8 \mathrm{H}), 0.90(\mathrm{t}, J=6.8 \mathrm{~Hz}, 3 \mathrm{H}) ;{ }^{13} \mathrm{C}$ NMR $\left(100 \mathrm{MHz}, \mathrm{CDCl}_{3}\right) \delta=$ 212.2, 91.9, 39.3, 33.6, 31.4, 28.8, 25.3, 22.4, 14.0; IR (neat) 2952, 2925, $2856,1184,1048 \mathrm{~cm}^{-1}$.

3c: ${ }^{[9]}$ Spectral data completely matched with reported data. ${ }^{1} \mathrm{H}$ NMR $(400$ $\left.\mathrm{MHz}, \mathrm{CDCl}_{3}\right): \delta=4.35-4.43(\mathrm{~m}, 1 \mathrm{H}), 3.96(\mathrm{dd}, J=5.2,11.8 \mathrm{~Hz}, 1 \mathrm{H}), 3.71$ $(\mathrm{dd}, J=8.0,12.0 \mathrm{~Hz}, 1 \mathrm{H}), 1.85-2.01(\mathrm{~m}, 2 \mathrm{H}), 1.24-1.47(\mathrm{~m}, 8 \mathrm{H}), 0.89(\mathrm{t}$, $J=7.0 \mathrm{~Hz}, 3 \mathrm{H}) ;{ }^{13} \mathrm{C} \mathrm{NMR}\left(100 \mathrm{MHz}, \mathrm{CDCl}_{3}\right) \delta=227.9,61.0,48.2,33.5$, $31.5,28.8,28.2,22.5,14.0$; IR (neat) 2953, 2025, 2853, 1457, 1062, 873 $\mathrm{cm}^{-1}$.

2d: ${ }^{[9]}$ Spectral data completely matched with reported data. ${ }^{1} \mathrm{H}$ NMR (400 $\left.\mathrm{MHz}, \mathrm{CDCl}_{3}\right): \delta=5.77-5.87(\mathrm{~m}, 1 \mathrm{H}), 5.06-5.16(\mathrm{~m}, 3 \mathrm{H}), 3.61(\mathrm{dd}, J=6.6$, $10.8 \mathrm{~Hz}, 1 \mathrm{H}), 3.42(\mathrm{dd}, J=9.8,11.0 \mathrm{~Hz}, 1 \mathrm{H}), 2.22-2.37(\mathrm{~m}, 2 \mathrm{H}), 2.11-$ $2.20(\mathrm{~m}, 1 \mathrm{H}), 1.87-1.95(\mathrm{~m}, 1 \mathrm{H}) ;{ }^{13} \mathrm{C}$ NMR $\left(100 \mathrm{MHz}, \mathrm{CDCl}_{3}\right) \delta=212.0$, 136.1, 116.3, 90.9, 39.2, 32.7, 29.4; IR (neat) 3076, 2977, 2935, 2849 $1640,1437,1350,1182,1047,914,847,647 \mathrm{~cm}^{-1}$

3d: ${ }^{1} \mathrm{H}$ NMR $\left(400 \mathrm{MHz}, \mathrm{CDCl}_{3}\right): \delta=5.73-5.83(\mathrm{~m}, 1 \mathrm{H}), 5.05-5.12(\mathrm{~m}, 2 \mathrm{H})$, $4.37-4.43(\mathrm{~m}, 1 \mathrm{H}), 3.99(\mathrm{dd}, J=5.4,11.8 \mathrm{~Hz}, 1 \mathrm{H}), 3.71$ (dd, $J=7.2,12.0$ $\mathrm{Hz}, 1 \mathrm{H}), 2.14-2.30(\mathrm{~m}, 2 \mathrm{H}), 1.96-2.14(\mathrm{~m}, 2 \mathrm{H}) ;{ }^{13} \mathrm{C}$ NMR $(100 \mathrm{MHz}$, $\left.\mathrm{CDCl}_{3}\right) \delta=227.5,136.1,116.7,59.9,48.1,32.6,32.1$; IR (neat) 3074 , 2976, 2920, 2848, 1639, 1444, 1434, 1421, 1059, 914, $870 \mathrm{~cm}^{-1}$; HRMS (FAB) $\mathrm{m} / \mathrm{z}$ calcd for $\mathrm{C}_{7} \mathrm{H}_{10} \mathrm{~S}_{3}: 189.9945\left(\left[\mathrm{M}^{+}\right]\right)$, found 189.9945.

2e: ${ }^{[9]}$ Spectral data completely matched with reported data. ${ }^{1} \mathrm{H}$ NMR $(400$ $\left.\mathrm{MHz}, \mathrm{CDCl}_{3}\right): \delta=5.85-5.94(\mathrm{~m}, 1 \mathrm{H}), 5.21-5.34(\mathrm{~m}, 3 \mathrm{H}), 4.08-4.09(\mathrm{~m}$, $2 \mathrm{H}$ ), 3.82 (dd, $J=5.4,10.6 \mathrm{~Hz}, 1 \mathrm{H}$ ), 3.77 (dd, $J=4.4,10.8 \mathrm{~Hz}, 1 \mathrm{H}$ ), 3.70 (dd, $J=8.4,11.2 \mathrm{~Hz}, 1 \mathrm{H}), 3.62(\mathrm{dd}, J=7.2,10.8 \mathrm{~Hz}, 1 \mathrm{H}) ;{ }^{13} \mathrm{C}$ NMR $(100$ $\left.\mathrm{MHz}, \mathrm{CDCl}_{3}\right) \delta=211.9,133.5,117.7,89.1,72.4,68.3,35.9$; IR (neat) 3079 , $3010,2979,2921,2859,1345,1228,1183,1093,1043,924,843 \mathrm{~cm}^{-1}$

3e: ${ }^{[9]}$ Spectral data completely matched with reported data. ${ }^{1} \mathrm{H}$ NMR $(400$ $\left.\mathrm{MHz}, \mathrm{CDCl}_{3}\right): \delta=5.85-5.95(\mathrm{~m}, 1 \mathrm{H}), 5.22-5.34(\mathrm{~m}, 2 \mathrm{H}), 4.44-4.50(\mathrm{~m}$, $1 \mathrm{H}), 4.09(\mathrm{dd}, J=5.8,8.4 \mathrm{~Hz}, 1 \mathrm{H}), 4.04-4.07(\mathrm{~m}, 2 \mathrm{H}), 3.97(\mathrm{dd}, J=4.4$, $12.4 \mathrm{~Hz}, 1 \mathrm{H}), 3.84(\mathrm{dd}, J=9.2,10.0 \mathrm{~Hz}, 1 \mathrm{H}), 3.65(\mathrm{dd}, J=5.8,9.8 \mathrm{~Hz}$, $1 \mathrm{H}) ;{ }^{13} \mathrm{C} \mathrm{NMR}\left(100 \mathrm{MHz}, \mathrm{CDCl}_{3}\right) \delta=227.2,133.8,118.0,72.4,69.0,58.1$, 44.9; IR (neat) 2924, 2853, 1421, 1265, 1068, 1035, 997, 928, 864, 735 $\mathrm{cm}^{-1}$.

2f: ${ }^{[9]}$ Spectral data completely matched with reported data. ${ }^{1} \mathrm{H}$ NMR (400 $\left.\mathrm{MHz}, \mathrm{CDCl}_{3}\right): \delta=7.30-7.40(\mathrm{~m}, 5 \mathrm{H}), 5.21-5.28(\mathrm{~m}, 1 \mathrm{H}), 4.64(\mathrm{~d}, J=11.6$ $\mathrm{Hz}, 1 \mathrm{H}), 4.60(\mathrm{~d}, J=12.0 \mathrm{~Hz}, 1 \mathrm{H}), 3.84(\mathrm{dd}, J=5.0,10.6 \mathrm{~Hz}, 1 \mathrm{H}), 3.78$ (dd, $J=4.6,10.6 \mathrm{~Hz}, 1 \mathrm{H}$ ), 3.69 (dd, $J=8.4,10.8 \mathrm{~Hz}, 1 \mathrm{H}$ ), 3.60 (dd, $J=$ 7.2, $11.2 \mathrm{~Hz}, 1 \mathrm{H}) ;{ }^{13} \mathrm{C}$ NMR $\left(100 \mathrm{MHz}, \mathrm{CDCl}_{3}\right) \delta=211.8,137.0,128.5$, 128.0, 127.7, 89.0, 73.7, 68.4, 36.0; IR (neat) 2922, 2863, 1731, 1453, $1343,1228,1185,1095,1038,913,848,737,697 \mathrm{~cm}^{-1}$.

3f: ${ }^{[9]}$ Spectral data completely matched with reported data. ${ }^{1} \mathrm{H}$ NMR (400 $\left.\mathrm{MHz}, \mathrm{CDCl}_{3}\right): \delta=7.30-7.40(\mathrm{~m}, 5 \mathrm{H}), 4.59(\mathrm{~s}, 2 \mathrm{H}), 4.44-4.50(\mathrm{~m}, 1 \mathrm{H}), 4.07$ (dd, $J=6.0,12.4 \mathrm{~Hz}, 1 \mathrm{H}$ ), 3.95 (dd, $J=4.8,12.0 \mathrm{~Hz}, 1 \mathrm{H}$ ), 3.85 (dd, $J=$ 9.2, $9.4 \mathrm{~Hz}, 1 \mathrm{H}), 3.69$ (dd, $J=5.8,9.8 \mathrm{~Hz}, 1 \mathrm{H}) ;{ }^{13} \mathrm{C} \mathrm{NMR}\left(100 \mathrm{MHz}, \mathrm{CDCl}_{3}\right)$ $\delta=227.2,137.2,128.6,128.1,127.7,73.5,69.0,58.2,44.9$; IR (neat) 2923, 2855, 1453, 1361, 1068, 1034, 864, 736, $696 \mathrm{~cm}^{-1}$.

2g: $:^{[9]}$ Spectral data completely matched with reported data. ${ }^{1} \mathrm{H}$ NMR (400 $\left.\mathrm{MHz}, \mathrm{CDCl}_{3}\right): \delta=5.35-5.42(\mathrm{~m}, 1 \mathrm{H}), 3.85-3.96(\mathrm{~m}, 2 \mathrm{H}), 3.70-3.82(\mathrm{~m}$, $2 \mathrm{H}) ;{ }^{13} \mathrm{C}$ NMR $\left(100 \mathrm{MHz}, \mathrm{CDCl}_{3}\right) \delta=210.7,88.2,42.7,36.7$; IR (neat) $3047,2954,1438,1428,1338,1265,1179,1038,732,701 \mathrm{~cm}^{-1}$. 
(S)-2h: HPLC analysis: Daicel Chiralcel OD-3, haxane/2-propanol $=2: 1$, flow rate $=0.5 \mathrm{~mL} / \mathrm{min}, 254 \mathrm{~nm}$; retention time: $37.0 \mathrm{~min}$ (minor) and 40.1 $\min$ (major). $[\alpha]^{28} \mathrm{D}=-57.6\left(c=1.0, \mathrm{CHCl}_{3} ;>99 \%\right.$ ee). ${ }^{1} \mathrm{H} \mathrm{NMR}(400 \mathrm{MHz}$, $\left.\mathrm{CDCl}_{3}\right): \delta=7.88-7.91(\mathrm{~m}, 2 \mathrm{H}), 7.76-7.80(\mathrm{~m}, 2 \mathrm{H}), 5.41-5.48(\mathrm{~m}, 1 \mathrm{H}), 4.27$ (dd, $J=6.8,14.0 \mathrm{~Hz}, 1 \mathrm{H}$ ), 4.06 (dd, $J=5.4,14.2 \mathrm{~Hz}, 1 \mathrm{H}$ ), 3.75 (dd, $J=$ 7.6, $11.2 \mathrm{~Hz}, 1 \mathrm{H}$ ), 3.60 (dd, $J=7.0,11.4 \mathrm{~Hz}, 1 \mathrm{H}) ;{ }^{13} \mathrm{C}$ NMR $(100 \mathrm{MHz}$, $\left.\mathrm{CDCl}_{3}\right) \delta=210.3,167.7,134.5,131.6,123.7,86.6,39.5,37.3$; IR (neat) 3062, 3027, 2997, 2952, 1773, 1704, 1414, 1393, 1370, 1309, 1238, 1167, 1055, 1034, 1004, 957, 721, $712 \mathrm{~cm}^{-1}$; HRMS (FAB) $\mathrm{m} / \mathrm{z}$ calcd for $\mathrm{C}_{12} \mathrm{H}_{9} \mathrm{NO}_{3} \mathrm{~S}_{2}: 279.0024\left(\left[\mathrm{M}^{+}\right]\right)$, found 279.0007.

$(R)$-3h: HPLC analysis: Daicel Chiralcel OD-3, haxane/2-propanol $=2: 1$, flow rate $=0.5 \mathrm{~mL} / \mathrm{min}, 254 \mathrm{~nm}$; retention time: $33.2 \mathrm{~min}$ (major) and 43.1 $\min$ (minor). $[\alpha]^{20} \mathrm{D}=+193.2\left(c=1.0, \mathrm{CHCl}_{3} ; 99 \%\right.$ ee $)$. Spectral data completely matched with reported data. ${ }^{[9]}{ }^{1} \mathrm{H} \mathrm{NMR}\left(400 \mathrm{MHz}, \mathrm{CDCl}_{3}\right): \delta=$ 7.89-7.92 (m, 2H), 7.77-7.81 (m, 2H), 4.64-4.71 (m, 1H), $4.28(\mathrm{dd}, J=$ 8.4, $14.0 \mathrm{~Hz}, 1 \mathrm{H}$ ), 4.12 (dd, $J=5.2,12.0 \mathrm{~Hz}, 1 \mathrm{H}$ ), 4.08 (dd, $J=5.6,14.0$ $\mathrm{Hz}, 1 \mathrm{H}), 3.77$ (dd, $J=4.4,12.4 \mathrm{~Hz}, 1 \mathrm{H}) ;{ }^{13} \mathrm{C}$ NMR $\left(100 \mathrm{MHz}, \mathrm{CDCl}_{3}\right) \delta=$ 225.6, 168.0, 134.5, 131.6, 123.8, 57.2, 45.9, 39.2; IR (neat) 3058, 2979, 2929, 1770, 1706, 1424, 1391, 1359, 1098, 1065, $715 \mathrm{~cm}^{-1}$.

6: ${ }^{1} \mathrm{H}$ NMR $\left(400 \mathrm{MHz}, \mathrm{CDCl}_{3}\right): \delta=3.90(\mathrm{~d}, J=12.0 \mathrm{~Hz}, 1 \mathrm{H}), 3.84(\mathrm{~d}, J=$ $11.6 \mathrm{~Hz}, 1 \mathrm{H}), 3.69(\mathrm{~d}, J=12.0 \mathrm{~Hz}, 1 \mathrm{H}), 3.44,(\mathrm{~d}, J=11.2 \mathrm{~Hz}, 1 \mathrm{H}), 1.77(\mathrm{~s}$, $3 \mathrm{H}) ;{ }^{13} \mathrm{C} \mathrm{NMR}\left(100 \mathrm{MHz}, \mathrm{CDCl}_{3}\right) \delta=210.0,96.5,47.3,41.5,22.9$; IR (neat) 2982, 2951, 2931, 2853, 1436, 1381, 1268, 1197, 1166, 1107, 1058, 1037, $854,792,745 \mathrm{~cm}^{-1}$; HRMS (FAB) $\mathrm{m} / \mathrm{z}$ calcd for $\mathrm{C}_{5} \mathrm{H}_{7} \mathrm{ClOS}_{2}: 181.9627$ $\left(\left[\mathrm{M}^{+}\right]\right)$, found 181.9627 .

trans-9:[9] Spectral data completely matched with reported data. ${ }^{1} \mathrm{H}$ NMR $\left(400 \mathrm{MHz}, \mathrm{CDCl}_{3}\right): \delta=4.35$ (td, $\left.J=4.0,11.6 \mathrm{~Hz}, 1 \mathrm{H}\right), 3.73(\mathrm{td}, J=4.0$, $12.0 \mathrm{~Hz}, 1 \mathrm{H}), 2.41-2.47(\mathrm{~m}, 1 \mathrm{H}), 2.17-2.23(\mathrm{~m}, 1 \mathrm{H}), 1.97-2.03(\mathrm{~m}, 1 \mathrm{H})$, 1.88-1.94 (m, 1H), 1.72-1.82 (m, 1H), 1.58-1.69 (m, 1H), 1.32-1.53 (m, $2 \mathrm{H}) ;{ }^{13} \mathrm{C}$ NMR $\left(100 \mathrm{MHz}, \mathrm{CDCl}_{3}\right) \delta=212.3,94.6,56.3,29.7,28.1,25.0$, 23.6; IR (neat) 2941, 2862, 1454, 1444, 1266, 1254, 1214, 1206, 1179 , $1146,1084,1058,1038,1000,959,902,862,833,637,614 \mathrm{~cm}^{-1}$.

trans-10:[9] Spectral data completely matched with reported data. ${ }^{1} \mathrm{H}$ NMR $\left(400 \mathrm{MHz}, \mathrm{CDCl}_{3}\right): \delta=4.05-4.14(\mathrm{~m}, 2 \mathrm{H}), 2.20-2.24(\mathrm{~m}, 2 \mathrm{H}), 1.91-2.01$ $(\mathrm{m}, 2 \mathrm{H}), 1.68-1.78(\mathrm{~m}, 2 \mathrm{H}), 1.40-1.53(\mathrm{~m}, 2 \mathrm{H}) ;{ }^{13} \mathrm{C} \mathrm{NMR}(100 \mathrm{MHz}$, $\left.\mathrm{CDCl}_{3}\right) \delta=227.1,64.4,29.0,25.0$; IR (neat) 2939, 2856, 1445, 1441, 1280 $1265,1100,1058,1035,863,740 \mathrm{~cm}^{-1}$

\section{Acknowledgements}

This work was partially supported by a Grant-in-Aid for Scientific Research (C) from JSPS, the Cooperative Research Program of "Network Joint Research Center for Materials and Devices", the Tokyo Kasei Chemical Promotion Foundation, and the Asahi Glass Foundation.

Keywords: alkali metals $•$ carbonates $\cdot$ green chemistry • reaction mechanisms $\cdot$ sustainable chemistry

[1] For reviews on green and sustainable chemistry, see: a) S. L. Y. Tang, R. L. Smith, M. Poliakoff, Green Chem. 2005, 7, 761-762; b) P. Anastas N. Eghbali, Chem. Soc. Rev. 2010, 39, 301-312.

[2] For reviews on crown ethers and polyethylene glycols, see: a) C. J. Pedersen, H. K. Frensdorff, Angew. Chem. 1972, 84, 16-26; Angew. Chem. Int. Ed. Engl. 1972, 11, 16-25; b) G. W. Gokel, H. D. Durst Synthesis 1976, 168-184; c) F. Vögtle, E. Weber, Angew. Chem. 1979,
91, 813-837; Angew. Chem. Int. Ed. Engl. 1979, 18, 753-776; d) C. J. Pedersen, Angew. Chem. 1988, 100, 1053-1059; Angew. Chem. Int. Ed. Engl. 1988, 27, 1021-1027; e) R. M. Izatt, K. Pawlak, J. S. Bradshaw, Chem. Rev. 1991, 91, 1721-2085; f) T. Okada, Analyst 1993, 118, 959971; g) R. D. Hancock, J. Chem. Educ. 1992, 69, 615-621; h) R. D. Hancock, J. Incl. Phenom. Mol. Recog. Chem. 1994, 17, 63-80; i) J. S. Bradshaw, R. M. Izatt, Acc. Chem. Res. 1997, 30, 338-345.

[3] For reviews on $\mathrm{CO}_{2}$ fixation reactions with epoxides, see: a) D. J. Darensbourg, M. W. Holtcamp, Coord. Chem. Rev. 1996, 153, 155-174; b) A. Decortes, A. M. Castilla, A. W. Kleij, Angew. Chem. 2010, 122 10016-10032; Angew. Chem. Int. Ed. 2010, 49, 9822-9837; c) M. North, R. Pasquale, C. Young, Green Chem. 2010, 12, 1514-1539; d) B.-H. Xu, J.-Q. Wang, J. Sun, Y. Huang, J.-P. Zhang, X.-P. Zhang, S.-J. Zhang, Green Chem. 2015, 17, 108-122; e) J. W. Comerford, I. D. V. Ingram, M. North, X. Wu, Green Chem. 2015, 17, 1966-1987; f) M. Cokoja, M. E. Wilhelm, M. H. Anthofer, W. A. Herrmann, F. E. Kühn, ChemSusChem 2015, 8, 2436-2454; g) C. Martín, G. Fiorani, A. W. Kleij, ACS Catal. 2015, 5, 1353-1370; h) H. Büttner, L. Longwitz, J. Steinbauer, C. Wulf, T. Werner, Top. Curr. Chem. 2017, 375, 50; i) M. Alves, B. Grignard, R. Mereau, C. Jerome, T. Tassaing, C. Detrembleur, Catal. Sci. Technol. 2017, 7, 2651-2684; j) R. R. Shaikh, S. Pornpraprom, V. D'Elia, ACS Catal. 2018, 8, 419-450.

[4] For examples of synthesis of cyclic carbonates using KI, see: a) $\mathrm{H}$. Zhu, L.-B. Chen, Y.-Y. Jiang, Polym. Adv. Technol. 1996, 7, 701-703; b) J. Song, Z. Zhang, B. Han, S. Hu, W. Li, Y. Xie, Green Chem. 2008, 10, 1337-1341; c) S. Liang, H. Liu, T. Jiang, J. Song, G. Yang, B. Han, Chem. Commun. 2011, 47, 2131-2133; d) J. Ma, J. Song, H. Liu, J. Liu, Z. Zhang, T. Jiang, H. Fan, B. Han, Green Chem. 2012, 14, 1743-1748; e) J. Ma, J. Liu, Z. Zhang, B. Han, Green Chem. 2012, 14, 2410-2420; f) J. Shi, J. Song, J. Ma, Z. Zhang, H. Fan, B. Han, Pure Appl. Chem. 2013, 85, 1633-1641; g) Z. Wu, H. Xie, X. Yu, E. Liu, ChemCatChem 2013, 5, 1328-1333; h) J. Tharun, G. Mathai, A. C. Kathalikkattil, R. Roshan, J.Y. Kwak, D.-W. Park, Green Chem. 2013, 15, 1673-1677; i) Z. Yang, J. Sun, W. Cheng, J. Wang, Q. Li, S. Zheng, Catal. Commun. 2014, 44, 69; j) K. R. Roshan, A. C. Kathalikkattil, J. Tharun, D. W. Kim, Y. S. Won D. W. Park, Dalton Trans. 2014, 43, 2023-2031; k) M. E. Wilhelm, M. H. Anthofer, M. Cokoja, I. I. E. Markovits, W. A. Herrmann, F. E. Kühn, ChemSusChem 2014, 7, 1357-1360; see also: I) B. Schäffner, M. Blug, D. Kruse, M. Polyakov, A. Köckritz, A. Martin, P. Rajagopalan, U. Bentrup, A. Brückner, S. Jung, D. Agar, B. Rüngeler, A. Pfennig, K. Müller, W. Arlt, B. Woldt, M. Graß, S. Buchholz, Chem SusChem 2014, 7, 1133-1139; m) S. Kumar, S. L. Jain, Ind. Eng. Chem. Res. 2014, 53, 541-546; n) J. Steinbauer, A. Spannenberg, T. Werner, Green Chem. 2017, 19, 37693779; о) J. Steinbauer, T. Werner, ChemSusChem 2017, 10, 30253029; p) L. Longwitz, J. Steinbauer, A. Spannenberg, T. Werner, ACS Catal. 2018, 8, 665-672.

[5] For examples of synthesis of cyclic carbonates with 18-crown-6 complex of KI, see: a) G. Rokicki, W. Kuran, B. Pogorzelska-Marciniak, Monatsh. Chem. 1984, 115, 205-214; b) N. Kihara, N. Hara, T. Endo, J. Org. Chem. 1993, 58, 6198-6202; c) K. Kasuga, N. Kabata, Inorg. Chim. Acta 1997, 257, 277-278; d) W. Desens, T. Werner, Adv. Synth. Catal. 2016, 358, 622-630.

[6] a) S. Liu, N. Suematsu, K. Maruoka, S. Shirakawa, Green Chem. 2016 18, 4611-4615; b) Y. Kumatabara, M. Okada, S. Shirakawa, ACS Sustainable Chem. Eng. 2017, 5, 7295-7301.

[7] For examples of synthesis of cyclic carbonates under mild reaction conditions, see: a) X.-B. Lu, B. Liang, Y.-J. Zhang, Y.-Z. Tian, Y.-M. Wang, C.-X. Bai, H. Wang, R. Zhang, J. Am. Chem. Soc. 2004, 126, 3732-3733; b) N. Aoyagi, Y. Furusho, T. Endo, Tetrahedron Lett. 2013, 54, 7031-7034; c) A. M. Hardman-Baldwin, A. E. Mattson, ChemSusChem 2014, 7, 3275-3278; d) H. Zhou, G.-X. Wang, W.-Z. Zhang, X.-B. Lu, ACS Catal. 2015, 5, 6773-6779; e) L. Wang, G. Zhang, K. Kodama, T. Hirose, Green Chem. 2016, 18, 1229-1233; f) J. A. Castro-Osma, M. North, X. Wu, Chem. Eur. J. 2016, 22, 2100-2107; g) J. A. Castro-Osma, K. J. Lamb, M. North, ACS Catal. 2016, 6, 5012- 
5025; h) M. Tiffner, S. Gonglach, M. Haas, W. Schöfberger, M. Waser, Chem. Asian J. 2017, 12, 1048-1051.

[8] S. Kaneko, S. Shirakawa, ACS Sustainable Chem. Eng. 2017, 5, 2836 2840 .

[9] For examples of synthesis of cyclic thiocarbonates with epoxides and $\mathrm{CS}_{2}$, see: a) Y. Taguchi, M. Yasumoto, I. Shibuya, Y. Suhara, Bull. Chem. Soc. Jpn. 1989, 62, 474-478; b) N. Kihara, Y. Nakawaki, T. Endo, J. Org. Chem. 1995, 60, 473-475; c) I. Yavari, M. Ghazanfarpour-Darjani, Z. Hossaini, M. Sabbaghan, N. Hosseini, Synlett 2008, 889-891; d) R. Maggi, C. Malmassari, C. Oro, R. Pela, G. Sartori, L. Soldi, Synthesis 2008, 53-56; e) A. Z. Halimehjani, F. Ebrahimi, N. Azizi, M. R. Saidi, J. Heterocycl. Chem. 2009, 46, 347-350; f) W. Clegg, R. W. Harrington, M. North, P. Villuendas, J. Org. Chem. 2010, 75, 6201-6207; g) C. Beattie, M. North, Chem. Eur. J. 2014, 20, 8182-8188; h) C. Beattie, M. North, ChemCatChem 2014, 6, 1252-1259; i) J. Cao, M. Yu, H. Li, L. Wang, X. Zhu, G. Wang, Y. Shi, C. Cao, Res. Chem. Intermed. 2015, 41, 5323-
5330; j) J. Diebler, A. Spannenberg, T. Werner, ChemCatChem 2016, 8 2027-2030; k) J. Diebler, A. Spannenberg, T. Werner, Org. Biomol. Chem. 2016, 14, 7480-7489; I) M. Ghazanfarpour-Darjani, A. Khodakarami, Monatsh. Chem. 2016, 147, 829-835; m) C. Mei, X. Li, L. Liu, C. Cao, G. Pang, Y. Shi, Tetrahedron 2017, 73, 5706-5714.

[10] The crystal structures of $(S)-2 h$ and $(R)-3 h$ have been deposited the Cambridge Crystallographic Data Centre (CCDC 1813513 for (S)-2h, CCDC 1813514 for $(R)-3 h)$.

[11] a) S. Malaschichin, C. Fu, A. Linden, H. Heimgartner, Helv. Chim. Acta 2005, 88, 3253-3262; b) S. Liao, M. Leutzsch, M. R. Monaco, B. List, J. Am. Chem. Soc. 2016, 138, 5230-5233.

[12] Y. Taguchi, K. Yanagiya, I. Shibuya, Y. Suhara, Bull. Chem. Soc. Jpn. 1987, 60, 727-730.

[13] W.-M. Ren, Y. Liu, X.-B. Lu, J. Org. Chem. 2014, 79, 9771-9777. 
WILEY-VCH

\section{FULL PAPER}

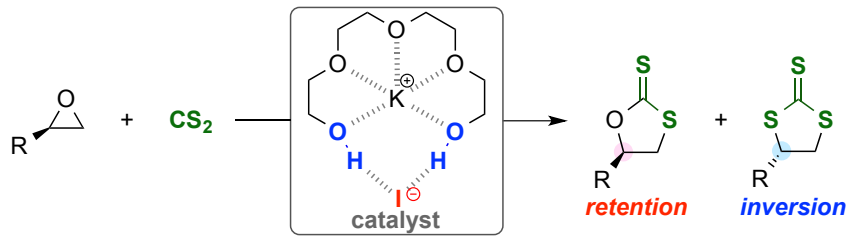

Efficient synthesis of cyclic thiocarbonates from epoxides and $\mathrm{CS}_{2}$ was achieved when a Kl-tetraethylene glycol complex was used as an economical catalyst. The mechanism for this reaction was discussed based on the stereochemistry of products.

\section{Sustainable Catalyst}

M. Okada, R. Nishiyori, S. Kaneko, K. Igawa, S. Shirakawa*

Page No. - Page No.

$\mathrm{KI}$-Tetraethylene Glycol Complex as an Effective Catalyst for the Synthesis of Cyclic Thiocarbonates from Epoxides and $\mathrm{CS}_{2}$ 